\title{
EFEITOS DE DOSES CRESCENTES DE CALCÁRIO NA PRODUÇÃO DE MUDAS DE SUMAÚMA (Ceiba pentandra L. Gaertn)
}

\author{
André Rogério Matos da Silva*, Carlos Alberto Franco Tucci**, Hedinaldo Narciso Lima**, \\ Patrícia Aparecida de Souza***, Nelson Venturin**** \\ *Eng. Florestal, M.Sc., Escola Agrotécnica Federal de São Gabriel da Cachoeira - arogerioms@hotmail.com \\ **Eng. Agrônomo, Dr., Faculdade de Ciências Agrárias, UFAM - ctucci@ufam.edu.br - hedinaldo@ufam.edu.br \\ ***Enga. Florestal Dra ., Depto. de Engenharia Agrícola e Solos, UFAM - patriciaapsouza@yahoo.com.br \\ ****Eng. Florestal, Dr., Depto. de Ciências Florestais, UFLA - venturim@ufla.br
}

Recebido para publicação: 21/07/2007 - Aceito para publicação: 23/10/2007

\begin{abstract}
A presente pesquisa foi realizada em viveiro na cidade de Manaus (AM), com o objetivo de avaliar os efeitos de doses crescentes de calcário sobre o crescimento e a absorção de nutrientes por mudas de sumaúma (Ceiba pentandra L. Gaertn). Como substrato, foi utilizado um material coletado no horizonte $\mathrm{B}$ de um Latossolo Amarelo álico muito argiloso. O calcário utilizado foi uma mistura de carbonato de cálcio e carbonato de magnésio p.a., na relação de 4:1. Os tratamentos foram doses crescentes de calcário e equivalentes a 0,$0 ; 0,5 ; 1,0 ; 2,0 ; 2,5 ; 3,0$ e 5,0 tha $^{-1}$. A fertilidade do substrato foi corrigida com a aplicação de $\mathrm{N}, \mathrm{P}_{2} \mathrm{O}^{5}$ e $\mathrm{K}_{2} \mathrm{O}$, e micronutrientes em doses equivalentes a 200,500 e $300 \mathrm{~kg}^{-h a^{-1}}$, respectivamente, mais $15 \mathrm{~kg} \cdot \mathrm{ha}^{-1}$ de FTE-Br12. Utilizou-se o delineamento experimental em blocos casualizados, com cinco repetições, num total de 35 parcelas. Cada parcela foi formada por três plantas. As características de crescimento avaliadas foram altura, diâmetro do colo, matéria seca da parte aérea, raiz e total, relação parte aérea/raiz. Os resultados mostraram que a correção do solo influenciou todas as características avaliadas, exceto a relação parte aérea/raiz. A exigência nutricional da espécie, na fase estudada, obedeceu à seguinte ordem decrescente $\mathrm{N}>\mathrm{K}>\mathrm{Ca}>\mathrm{Mg}>\mathrm{P}>\mathrm{Fe}>\mathrm{Mn}>\mathrm{Zn}>\mathrm{Cu}$. A dose de calcário que acusou o melhor rendimento econômico corresponde a 0,5 t.ha ${ }^{-1}$, ou $250 \mathrm{~g}$ de calcário por $1000 \mathrm{~kg}^{-1}$ de substrato, podendo ser esta a recomendada para subsolos ácidos e de baixa fertilidade natural.

Palavras-chave: Calagem; espécies florestais; Malvaceae; crescimento de mudas.
\end{abstract}

Resumo

\section{Abstract}

Effects of increasing doses of corrective in the formation of sumauma (Ceiba pentandra L. Gaertn), seedlings. This study was carried out in a nursery at Manaus, AM, in order to evaluate the effect of increasing levels of limimg on the growth and on the absorption of nutrients of sumaúma (Ceiba pentandra L. Gaertn) seedlings. Material collected in horizon B of clay yellow oxisol was used as substratum. The used calcareous rock was a carbonate mixture of calcium and magnesium carbonate p.a., in the relation of $4: 1$, proportion. The treatments were increasing liming levels, $0.0 ; 0.5 ; 1.0 ; 2.0$; $2.5 ; 3.0$ and 5.0 ton.ha $^{-1}$. The experimental design was randomized blocks with 5 replications in a total of 35 parcels, each one with 3 plants. The substratum was fertilized with equivalents doses to 200-500-300 kg.ha ${ }^{-1}$ of $\mathrm{N}, \mathrm{P}_{2} \mathrm{O}_{5}$ and $\mathrm{K}_{2} \mathrm{O}$, respectively and $15 \mathrm{~kg} \cdot \mathrm{ha}^{-1}$ of micronutrients FTE-Br12. The characteristics of growth evaluated were height, diameter of the stem, root, aerial part and total dry biomass and aerial part/root relation. It was determined the moisture contents of the aerial part, root and total. The liming affected all the growth characteristics, except the aerial part/root relation. The nutritional requirement, in the studied phase, followed the order of $\mathrm{N}>\mathrm{K}>\mathrm{Ca}>\mathrm{Mg}>\mathrm{P}>\mathrm{Fe}>\mathrm{Mn}>\mathrm{Zn}>\mathrm{Cu}$. The corrective dose of 0.5 t.ha ${ }^{-1}$ of soil or $250 \mathrm{~g}$ of corrective per ton of substratum was economically more advantageous and can be recommended to be used in acid subsoil of low natural fertility.

Keywords: Liming; seedling; Ceiba pentandra.

\section{INTRODUÇÃO}

A sumaúma é uma espécie que ocorre desde o México até o norte da América do Sul, incluindo a bacia amazônica, principalmente nas matas de várzea e de terra firme com solo argiloso (RIZZINI, 1990). Em ecossistemas de terra firme da Amazônia brasileira, onde, em geral, os solos são de baixa fertilidade natural, a espécie atinge menor porte, embora volumosa, enquanto que nos terrenos de várzeas inundáveis são maiores e com sapopemas (LOUREIRO et al., 1979). 
De acordo com Lamprecht (1990), a sumaúma apresenta maior crescimento em solos levemente ácidos, com valores de $\mathrm{pH}$ próximos à neutralidade. Essas características são mais comumente observadas nos solos de várzea da região, onde ocorre com maior freqüência e alcança maior e mais rápido crescimento.

Segundo Caliri et al. (2000), pouco se sabe sobre a exigência nutricional e crescimento de sumaúma em plantios, sejam eles homogêneos ou mistos. O mesmo acontece na fase de muda, pois existe grande dificuldade de se fazer recomendações de fertilização específicas para cada espécie (CRUZ et al., 2006). Todavia, alguns trabalhos têm sido desenvolvidos visando introduzir e acompanhar seu desenvolvimento, desde a fase inicial até a implantação em ecossistemas de terra firme com solos pobres quimicamente.

Em razão de suas características, a espécie é muito utilizada na indústria de chapas aglomeradas e de papel. Por isso, tem sido objeto de significativo extrativismo, o que pode levar a uma rápida redução do seu estoque. Alternativamente, a espécie pode ser cultivada em solos de terra firme, reduzindo dessa forma a pressão sobre os estoques naturais (TUCCI et al., 2004). Porém, características químicas dos solos de terra firme, tais como acidez elevada, alto teor de $\mathrm{Al}^{3+}$ trocável e deficiência de nutrientes, principalmente $\mathrm{Ca}, \mathrm{Mg}, \mathrm{P}$ e $\mathrm{K}$, podem constituir limitações ao cultivo e crescimento da sumaúma, que está mais bem adaptada aos solos de várzea, mais férteis e menos ácidos. Portanto, o uso de corretivos e fertilizantes é uma prática fundamental no processo de formação de mudas, não só para a redução da acidez do solo, mas também como fonte de nutrientes indispensáveis ao crescimento inicial das plantas, especialmente quando se utiliza, na preparação de substrato para produção de mudas, material de solos ácidos e pobres em nutrientes.

Nesse sentido, estudos que visem identificar os efeitos da correção da acidez do solo e do incremento de nutrientes sobre o desenvolvimento da sumaúma são indispensáveis e urgentes. Este trabalho foi realizado com o objetivo de avaliar os efeitos de doses crescentes de corretivo sobre o crescimento e a absorção de nutrientes por mudas de sumaúma.

\section{MATERIAL E MÉTODOS}

O experimento foi conduzido em Viveiro Florestal da Faculdade de Ciências Agrárias (FCA) em Manaus (AM), no período de setembro de 2002 a agosto de 2003. O material usado como substrato foi coletado no horizonte $\mathrm{Bw}$, que corresponde ao horizonte subsuperficial, de Latossolo Amarelo álico muito argiloso. O solo encontra-se sob a cobertura vegetal de uma floresta secundária com mais de 30 anos e está localizado no setor sul do campus universitário da Universidade Federal do Amazonas (UFAM), nas coordenadas UTM 21M0168944; 9637166.

Após a coleta, o material foi destorroado e passado em uma peneira com malha de $4 \mathrm{~mm}$ de abertura. Amostras desse material foram submetidas à análise química e à determinação da composição granulométrica, de acordo com procedimentos analíticos descritos por EMBRAPA (1997). A partir dos valores obtidos, calculou-se a saturação por bases, a capacidade de troca de cátions efetiva, a capacidade de troca de cátions total em pH 7,0 e a saturação por alumínio.

O delineamento experimental utilizado foi o de blocos casualizados, constituído de sete tratamentos e cinco repetições, num total de 35 parcelas, sendo cada parcela constituída de três plantas. Os tratamentos constituíram-se em doses crescentes de calcário e equivaleram a 0,$0 ; 0,5 ; 1,0 ; 2,0 ; 2,5 ; 3,0$ e 5,0 t.ha ${ }^{-1}$. O calcário utilizado foi uma mistura de carbonato de cálcio e carbonato de magnésio p.a., na relação $\mathrm{CaCO}_{3}: \mathrm{MgCO}_{3}$ de 4:1, com PRNT igual a 100\%. Após a aplicação do corretivo, o substrato ficou incubado por 30 dias, para que ocorresse a reação de neutralização da acidez.

Após correção da acidez, foi aplicado ao substrato superfosfato triplo e cloreto de potássio, em doses equivalentes a 500 e $300 \mathrm{~kg} \cdot \mathrm{ha}^{-1}$ de $\mathrm{P}_{2} \mathrm{O}_{5}$ e $\mathrm{K}_{2} \mathrm{O}$, respectivamente. Como fonte de micronutrientes, utilizou-se a FTE-Br12, em dose equivalente a $15 \mathrm{~kg} \cdot \mathrm{ha}^{-1}$. As doses foram selecionadas com base nas características do solo e em necessidades nutricionais de algumas espécies florestais (TUCCI et al., 2004). Após a aplicação dos fertilizantes, o substrato foi transferido para sacos plásticos com capacidade de $3,5 \mathrm{~kg}$. O adubo nitrogenado utilizado foi a uréia, aplicada na forma de solução e em cobertura 35 dias após a repicagem. A dose foi equivalente a $200 \mathrm{~kg} \cdot \mathrm{ha}^{-1}$ de $\mathrm{N}$.

As sementes de sumaúma foram submetidas à quebra de dormência por meio de submersão em água por 24 horas à temperatura ambiente. Foram colocadas para germinar em copos descartáveis de 300 
$\mathrm{ml}$ que continham areia lavada com solução de hipoclorito de sódio $0,1 \%$. As plântulas foram repicadas aos 28 dias, após germinação, quando mediam cerca de $15 \mathrm{~cm}$.

As mudas de sumaúma permaneceram em viveiro, com $40 \%$ de luminosidade. Foram regadas diariamente, e a umidade foi controlada por pesagem. $\mathrm{O}$ teor correspondeu à capacidade de campo para Latossolos, que é de cerca de 30\% do peso do solo seco, conforme sugerido por Tucci (1996).

Aos 97 dias após transplantio, as características de crescimento foram avaliadas por meio da tomada da altura da planta, medida da base até a gema apical, do diâmetro do colo (DC), da matéria seca da parte aérea (MSPA) e da raiz (MSR) e da relação raiz/parte aérea (PA/R), que foi calculada.

$\mathrm{O}$ material vegetal foi seco em estufa de circulação forçada a $70^{\circ} \mathrm{C}$, até atingir peso constante. Determinaram-se os teores totais de $\mathrm{N}, \mathrm{P}, \mathrm{K}, \mathrm{Ca}, \mathrm{Mg}, \mathrm{Fe}, \mathrm{Cu}, \mathrm{Zn}$ e $\mathrm{Mn}$. As análises foram realizadas no Laboratório de Solos da EMBRAPA-CPAA, segundo procedimento analítico descrito por Malavolta et al. (1989). Os conteúdos de N, P, K, Ca e Mg foram estimados a partir dos teores totais.

Não se conseguiu ajuste de modelo de regressão para as características avaliadas como função de doses de corretivo. Assim sendo, os resultados foram submetidos a teste de comparação de médias.

\section{RESULTADOS E DISCUSSÃO}

\section{Características químicas e composição granulométrica do substrato}

Os resultados da caracterização química e da composição granulométrica do material utilizado como substrato são: $\mathrm{pH}\left(\mathrm{H}_{2} \mathrm{O}\right)=5,5 ; \mathrm{Al}^{3+}=1,2 ; \mathrm{Ca}^{2+}+\mathrm{Mg}^{2+}=0,10 ; \mathrm{H}+\mathrm{Al}=6,35 ;$ CTC a pH 7,0 = 6,35 e CTC efetiva $=1,36$, expressos em $\mathrm{cmol}_{\mathrm{c}} \cdot \mathrm{kg}^{-1} ; \mathrm{P}=1 \mathrm{e} \mathrm{K}=24$, expressos em $\mathrm{mg} \cdot \mathrm{kg}^{-1}$; saturação por bases $(\mathrm{V})=1,5$ e saturação por alumínio $(\mathrm{m})=88$, expressos em $\%$; teor de matéria orgânica $=24,8$ e teor de argila $=798$, expressos em $\mathrm{g} \cdot \mathrm{kg}^{-1}$.

Esses resultados mostram um substrato com acidez ativa média, teores de $\mathrm{P}, \mathrm{Ca}$ e $\mathrm{Mg}$ muito baixos e baixo teor de K (CFSEMG, 1999), além de saturação por alumínio ( $\mathrm{m} \%$ ) muito elevada e baixa saturação por bases $(\mathrm{V} \%)$, como ocorre com a maioria dos solos bem drenados do ecossistema de terra firme da Amazônia brasileira.

\section{Crescimento das mudas}

As mudas de sumaúma responderam à aplicação de calcário em altura, diâmetro do colo, matéria seca da parte aérea e raiz e total (Tabela 1). A relação raiz/parte aérea não foi influenciada pela calagem. Essas respostas foram devido ao maior conteúdo de nutrientes nas mudas de sumaúma quando comparado com o das parcelas que não receberam o calcário (Tabela 2). Tucci et al. (2004) também observaram resposta positiva à calagem quando avaliaram os efeitos dessa prática sobre a altura, o diâmetro e a matéria seca de mudas de sumaúma. Diversas outras espécies arbóreas respondem à calagem, entre elas a cássia-verrugosa (Senna multijuga), o ipê-mirim (Stenolobium stans), o angico-do-cerrado (Anadenathera falcata), o cedro (Cedrela fissilis), segundo Furtini Neto et al. (1996), e pata-de-vaca (Bauhinia forficata Link), segundo Ramos (1999).

Por outro lado, Mann et al. (1996), estudando o crescimento da acácia (Acacia auriculiformes) e do jacarandá-branco (Platipodium elegans), concluíram que essas espécies não responderam à calagem, mostrando-se tolerantes à acidez. Entretanto, os mesmos autores encontraram respostas à calagem para outras seis espécies estudadas. Isso permite prever que as espécies florestais respondem de forma diferenciada à aplicação de calcário.

Respostas à adição de calcário também estão relacionadas à presença de outros nutrientes. Costa Filho (1986), ao avaliar os efeitos de níveis de calagem associados com diferentes níveis de $\mathrm{P}$ e $\mathrm{K}$ sobre o crescimento inicial de plantas de aroeira (Astronium urundeuva), concluiu que as doses de $\mathrm{P}$ e $\mathrm{K}$ na presença de calagem proporcionaram melhores respostas para o crescimento dessa espécie.

As diferenças em diâmetro do colo não foram consistentes, não permitindo nenhuma inferência (Tabela 1), apesar da análise de variância acusar diferença entre as médias dos tratamentos. Esses resultados indicam que, em doses elevadas, acima de 2,5 t.ha ${ }^{-1}$, o corretivo poderá causar redução do diâmetro (Tabela 1). Tucci et al. (2004) observaram que o diâmetro do colo de mudas de sumaúma aumentou com a aplicação de 2,03 t.ha ${ }^{-1}$ de corretivo quando comparado com a ausência de correção da acidez. 
Tabela 1. Crescimento em altura, diâmetro do colo, produção de matéria seca da parte aérea (MSPA), matéria seca da raiz (MSR), matéria seca total (MST) e relação raiz/parte aérea (R/PA) das plantas de sumaúma submetidas a doses crescentes de calcário aos 97 dias após transplantio das mudas (15 cm de altura) para sacos plásticos com 3,5 $\mathrm{kg}$ de substrato.

Table 1. Growth in height, diameter of the collar, production of dry matter of the aerial part (MSPA), dry matter of the root (MSR), total dry matter (MST) and relationship root/aerial part (R/PA) of the sumaúma plants submitted to growing doses of lime to the 97 days after transplanting of the seedlings ( $15 \mathrm{~cm}$ of height) to plastic bag with $3,5 \mathrm{~kg}$ of substratum.

\begin{tabular}{|c|c|c|c|c|c|c|}
\hline $\begin{array}{c}\text { Corretivo } \\
\left(\text { t.ha }^{-1}\right)\end{array}$ & $\begin{array}{l}\text { Altura } \\
\text { (cm) }\end{array}$ & $\begin{array}{c}\begin{array}{c}\text { Diâmetro do colo } \\
(\mathrm{mm})\end{array} \\
\end{array}$ & MSPA & $\begin{array}{l}\text { MSR } \\
\text { (g/ue) }\end{array}$ & MST & $\mathbf{P A} / \mathbf{R}$ \\
\hline 0,0 & $34,2 \mathrm{~b}$ & $6,00 \mathrm{ab}$ & $4,88 \mathrm{~b}$ & $1,74 \mathrm{~b}$ & $6,62 b$ & $2,93 \mathrm{a}$ \\
\hline 0,5 & $68,4 \mathrm{a}$ & $6,24 \mathrm{ab}$ & $32,75 \mathrm{a}$ & $10,14 \mathrm{a}$ & $42,89 \mathrm{a}$ & $3,34 \mathrm{a}$ \\
\hline 1,0 & $67,1 \mathrm{a}$ & $6,14 \mathrm{ab}$ & $27,91 \mathrm{a}$ & $8,81 \mathrm{a}$ & $36,72 \mathrm{a}$ & $3,09 \mathrm{a}$ \\
\hline 2,0 & $57,7 \mathrm{a}$ & $5,34 \mathrm{~b}$ & $19,26 \mathrm{ab}$ & $5,68 \mathrm{ab}$ & $24,94 \mathrm{ab}$ & $3,52 \mathrm{a}$ \\
\hline 2,5 & $67,5 \mathrm{a}$ & $6,72 \mathrm{a}$ & $31,60 \mathrm{a}$ & $10,49 \mathrm{a}$ & $42,09 \mathrm{a}$ & $3,19 \mathrm{a}$ \\
\hline 3,0 & $55,8 \mathrm{ab}$ & $5,74 \mathrm{~b}$ & $28,94 \mathrm{a}$ & $8,80 \mathrm{a}$ & $37,74 \mathrm{a}$ & $3,34 \mathrm{a}$ \\
\hline 5,0 & $69,4 \mathrm{a}$ & $5,82 \mathrm{ab}$ & $32,29 \mathrm{a}$ & $9,79 \mathrm{a}$ & $42,09 \mathrm{a}$ & $3,33 \mathrm{a}$ \\
\hline
\end{tabular}
experimental.

Tabela 2. Conteúdo total dos macronutrientes em mudas de sumaúma, 97 dias após transplantio das mudas ( $15 \mathrm{~cm}$ de altura) para sacos plásticos com 3,5 kg de substrato.

Table 2. Macronutrients total content in Ceiba pentrandra L. Gaertn seedlings, 97 days after transplanting of the seedlings ( $15 \mathrm{~cm}$ of height) to plastic bag with $3,5 \mathrm{~kg}$ of substratum.

\begin{tabular}{cccccc}
\hline $\begin{array}{c}\text { Corretivo } \\
\text { (t.ha }^{-1} \text { ) }\end{array}$ & $\mathbf{N}$ & $\mathbf{P}$ & $\mathbf{K}$ & $\mathbf{C a}$ & $\mathbf{M g}$ \\
\hline 0,0 & $12,23 \mathrm{~b}$ & $0,97 \mathrm{~b}$ & $7,91 \mathrm{~b}$ & $5,29 \mathrm{c}$ & $1,39 \mathrm{c}$ \\
0,5 & $92,85 \mathrm{a}$ & $10,81 \mathrm{a}$ & $86,47 \mathrm{a}$ & $39,82 \mathrm{ab}$ & $7,90 \mathrm{ab}$ \\
1,0 & $80,57 \mathrm{a}$ & $9,19 \mathrm{a}$ & $73,83 \mathrm{a}$ & $35,84 \mathrm{ab}$ & $7,72 \mathrm{ab}$ \\
2,0 & $58,15 \mathrm{ab}$ & $6,49 \mathrm{a}$ & $53,35 \mathrm{a}$ & $24,71 \mathrm{bc}$ & $5,61 \mathrm{bc}$ \\
2,5 & $89,14 \mathrm{a}$ & $9,93 \mathrm{a}$ & $77,59 \mathrm{a}$ & $46,39 \mathrm{ab}$ & $10,73 \mathrm{a}$ \\
3,0 & $77,32 \mathrm{a}$ & $9,58 \mathrm{a}$ & $73,49 \mathrm{a}$ & $43,92 \mathrm{ab}$ & $9,92 \mathrm{ab}$ \\
5,0 & $85,79 \mathrm{a}$ & $9,76 \mathrm{a}$ & $76,54 \mathrm{a}$ & $52,07 \mathrm{a}$ & $12,22 \mathrm{a}$ \\
\hline
\end{tabular}

Valores seguidos de letras distintas na mesma coluna diferem entre si pelo teste de Tukey a $5 \%$ de probabilidade. ue $=$ unidade experimental.

Com a aplicação de 0,5 t.ha $^{-1}$ de calcário, observou-se maior produção de matéria seca da parte aérea, da raiz e total, quando comparada à ausência de aplicação (Tabela 1). Entretanto, a dose 0,5 t.ha $^{-1}$ de calcário não diferiu estatisticamente das demais aplicadas, o que, do ponto de vista econômico, pode ser considerada a mais vantajosa.

A relação raiz/parte aérea (R/PA) apresentou valores estatisticamente equivalentes entre todos os tratamentos, inclusive na ausência de calagem (Tabela 1). Pode-se supor que as alterações ocasionadas pela calagem tragam efeitos semelhantes nos tecidos da parte aérea e das raízes.

Segundo Gonçalves et al. (2000), o estado nutricional das mudas está relacionado com a taxa de crescimento delas. A produção relativa de matéria seca total para as doses 0,$5 ; 1,0 ; 2,0 ; 2,5 ; 3,0$ e 5,0 foi de $648,554,377,635,570$ e $635 \%$, respectivamente, em relação à testemunha, tomada como referencial e considerada como $100 \%$, evidenciado os efeitos positivos da correção do substrato na formação de mudas de sumaúma. Essa diferença de comportamento demonstra que a sumaúma é uma espécie que possui uma elevada taxa de crescimento em fase de muda, o que permite inferir que a espécie possa ter um elevado requerimento nutricional nessa fase.

\section{Absorção de nutrientes}


Observou-se resposta positiva à aplicação da calagem, o que é evidenciado por conteúdos totais de nitrogênio, fósforo, potássio, cálcio e magnésio superiores na presença de corretivo, em comparação com sua ausência (Tabela 2).

A calagem favoreceu a absorção de nitrogênio, pois, na sua presença, o conteúdo de nitrogênio foi superior àquele observado na sua ausência, o que pode ser atribuído ao aumento do pH do solo e à adição de $\mathrm{Ca}$ e $\mathrm{Mg}$, favorecendo o aumento da atividade microbiana e a decomposição da matéria orgânica, com conseqüente aumento na mineralização do nitrogênio (VITTI et al., 1984). Apenas a aplicação de 2,0 t.ha ${ }^{-1}$ de corretivo não apresentou valor superior à ausência desta, o que pode ser atribuído ao baixo valor de matéria seca total (Tabela 1).

Houve resposta positiva da sumaúma quanto à absorção de fósforo, pois, quando se realizou a calagem, independentemente da dose aplicada, o conteúdo foi superior ao da testemunha (Tabela 2), o que sugere uma maior disponibilidade de fósforo no solo após a correção. Estudos sobre o efeito da calagem na disponibilidade de fósforo têm apresentado resultados variáveis. Segundo Novais et al. (1994), a calagem, juntamente com a fertilização fosfatada em fontes solúveis, causa aumento na disponibilidade de fósforo, e isso tem sido atribuído à maior hidrólise dos fosfatos de ferro e de alumínio, que os torna mais solúveis, e à redução na fixação de fósforo com o aumento de $\mathrm{pH}$. Espécies pioneiras, como é o caso da sumaúma, mostram-se responsivas ao fósforo, como comprovado em trabalhos realizados por Resende (1997) e Gomes (2004). Tucci et al. (2004) observaram interações positivas entre calagem e adubação fosfatada para a produção de mudas de sumaúma.

Utilizando-se calcário com relação Ca:Mg de 2:1 na produção de mudas de acácia-negra (Acacia mearnsii De Wild.), Tedesco (1999) verificou que a interação entre nitrogênio e fósforo foi significativa para as variáveis matéria seca aérea, matéria seca da raiz e matéria seca total. Segundo esse autor, as doses de nitrogênio que permitiram os melhores resultados variaram no intervalo de 0,66 a 2,32 g.planta ${ }^{-1}$, e as doses de fósforo, entre 2,51 e 6,27 g.planta ${ }^{-1}$. Esses resultados demonstram que há uma interação entre calagem e adubações nitrogenadas e fosfatadas, também observado por Tucci et al. (2004) estudando mudas de sumaúma (Ceiba pentandra).

A absorção de potássio pelas mudas de sumaúma também foi favorecida pela calagem, pois o conteúdo de potássio em todos os tratamentos com calagem superou aqueles em que não foi adicionado o corretivo (Tabela 2). Respostas positivas à absorção de potássio têm sido observadas por outros autores. Sousa (2000) observou que a relação cálcio/potássio influenciou nos aspectos nutricionais referentes ao acúmulo de nutrientes na matéria seca da raiz, na parte aérea, no acúmulo total e na translocação de nutrientes em mudas de açaizeiro (Euterpe oleraceae Mart.). Tucci et al. (2004) observaram interação negativa entre calagem e adubação potássica em mudas de sumaúma (Ceiba pentandra). Os autores sugeriram interação negativa entre $\mathrm{Ca}, \mathrm{Mg}$ e $\mathrm{K}$, utilizando-se dose superior à utilizada como corretiva da fertilidade do solo neste ensaio. Os resultados indicam a necessidade de estudos mais aprofundados no que diz respeito aos efeitos da interação $\mathrm{K} / \mathrm{Ca}$ e/ou $\mathrm{K} / \mathrm{Ca}+\mathrm{Mg}$ em mudas de sumaúma.

De modo geral, a calagem favoreceu significativamente a absorção de cálcio e magnésio (Tabela 2), o que se atribui ao aumento dos teores trocáveis desses nutrientes. Esses resultados diferem dos encontrados por Duboc (1994), que descreve um antagonismo entre a absorção de cálcio e magnésio, ou seja, o aumento da concentração de um desses elementos no meio implica a diminuição da absorção do outro, o que não ocorreu neste trabalho.

A tabela 3 demonstra que houve uma redução na absorção de cobre em função da calagem, concordando com Malavolta (1981), Quaggio (1986) e Osaki (1991), que mencionam que há redução na disponibilidade dos micronutrientes catiônicos com o aumento do $\mathrm{pH}$ do solo devido a aumento na precipitação. Por outro lado, os teores de manganês aumentaram com as doses crescentes de corretivo, ao contrário do esperado pela literatura (Tabela 3), porém semelhantes aos encontrados por Silva et al. (2007) trabalhando com mudas de mogno (Swietenia macrophyla King).

A calagem não influenciou a absorção de ferro e zinco (Tabela 3). Esperava-se uma redução na absorção com o aumento das doses de corretivo, pois quanto maior o valor do $\mathrm{pH}$ maior será a possibilidade de precipitação desses micronutrientes. Esse fato sugere que o aumento do $\mathrm{pH}$ não foi suficiente para reduzir a disponibilidade de ferro e manganês para as mudas de sumaúma. Também não se constatou deficiência desses nutrientes. 
Tabela 3. Conteúdo de micronutrientes na matéria seca da parte aérea em mudas de sumaúma, 97 dias após transplantio das mudas (15 cm de altura) para sacos plásticos com 3,5 kg de substrato.

Table 3. Micronutrient contents in dry biomass of the aerial part of sumauma seedlings, 97 days after transplanting of the seedlings ( $15 \mathrm{~cm}$ of height) for plastic bag with $3,5 \mathrm{~kg}$ of substratum.

\begin{tabular}{ccccc}
\hline $\begin{array}{c}\text { Corretivo } \\
\left(\mathbf{t} \cdot \mathbf{h a}^{-\mathbf{1}}\right)\end{array}$ & $\mathbf{C u}$ & $\mathbf{F e}$ & $\mathbf{M n}$ & $\mathbf{Z n}$ \\
\hline 0,0 & $11,8 \mathrm{a}$ & $259 \mathrm{a}$ & $28 \mathrm{c}$ & $39 \mathrm{a}$ \\
0,5 & $7,0 \mathrm{~b}$ & $281 \mathrm{a}$ & $44 \mathrm{ab}$ & $32 \mathrm{a}$ \\
1,0 & $6,6 \mathrm{~b}$ & $256^{\mathrm{a}}$ & $51 \mathrm{ab}$ & $32^{\mathrm{a}}$ \\
2,0 & $6,2 \mathrm{~b}$ & $231^{\mathrm{a}}$ & $40 \mathrm{bc}$ & $32^{\mathrm{a}}$ \\
2,5 & $5,8 \mathrm{~b}$ & $266^{\mathrm{a}}$ & $46 \mathrm{ab}$ & $31^{\mathrm{a}}$ \\
3,0 & $7,8 \mathrm{~b}$ & $250^{\mathrm{a}}$ & $54 \mathrm{ab}$ & $33^{\mathrm{a}}$ \\
5,0 & $7,2 \mathrm{~b}$ & $239^{\mathrm{a}}$ & $55 \mathrm{a}$ & $31 \mathrm{a}$ \\
\hline
\end{tabular}

Valores seguidos de letras distintas na mesma coluna diferem entre si pelo teste de Tukey a 5\% de probabilidade. ue $=$ unidade experimental.

\section{CONCLUSÕES}

A correção do solo favoreceu todas as características de crescimento avaliadas, exceto a relação R/PA.

A correção do solo influenciou positivamente a absorção de $\mathrm{N}, \mathrm{P}, \mathrm{K}, \mathrm{Ca}, \mathrm{Mg}$ e $\mathrm{Mn}$, negativamente a de $\mathrm{Cu}$ e não alterou a de $\mathrm{Fe}$ e $\mathrm{Zn}$.

A exigência nutricional da espécie obedeceu à seguinte ordem decrescente: $\mathrm{N}>\mathrm{K}>\mathrm{Ca}>\mathrm{Mg}>\mathrm{P}>\mathrm{Fe}>\mathrm{Mn}>\mathrm{Zn}>\mathrm{Cu}$.

A primeira recomendação de calagem para essa espécie, quando se utiliza subsolo ácido e de baixa fertilidade natural, deve ser de $0,5 \mathrm{t}^{-h a^{-1}}$, ou $0,250 \mathrm{~kg}$ de calcário com $100 \%$ de PRNT para cada $1000 \mathrm{~kg}$ de substrato.

\section{REFERÊNCIAS}

CALIRI, G. J. A.; AZEVEDO, C. P.; ROSSI, L. M. B.; VAN LEEUWEN, J. Caracterização do crescimento de sumaúma (Ceiba pentandra) sob diversas condições de plantio na Amazônia Central. In. CONGRESSO BRASILEIRO DE SISTEMAS AGROFLORESTAIS: MANEJANDO A BIODIVERSIDADE E COMPONDO A PAISAGEM RURAL, 3. 2000, Manaus. Anais... Manaus: Embrapa Amazônia Ocidental, 2000. 416 p.

COSTA FILHO, R. T. C. Crescimento de mudas de aroeira (Astronium urundeuva (Fr.all.) Engl.) em resposta a calagem, fósforo e potássio. 54 f. Dissertação (Mestrado em Ciência Florestal) Universidade Federal de Lavras, Lavras, 1986.

CRUZ, A. F.; PAIVA, H. N.; GUERREIRO, C. R. A. Efeito da adubação nitrogenada na produção de mudas de sete-cascos (Samanea inopinata (Harms) Ducke). Revista Árvore, Viçosa, MG, v. 30, n. 4, p. 537-546, 2006.

DUBOC, E. Requerimentos nutricionais de espécies nativas: Hymenaea courbaril L. var. Stilbocarpa (Hayne) Lee et Lang. (Jatobá), Copaifera langsdorffii Desf. (Óleo de copaíba) e Peltophorum dubium (Spreng) Taub. (Canafístula). 68 f. Dissertação (Mestrado em Solos) - Universidade Federal de Lavras, Lavras, 1994.

EMBRAPA. Serviço Nacional de Levantamento e Conservação de Solos. Manual de métodos de análise de solos. 2. ed. Rio de Janeiro, 1997.212 p.

FURTINI NETO, A. E.; ROSSI, A. R; RESENDE, A. V.; VALE, F. R. Acidez do solo e crescimento inicial de espécies florestais nativas. In.: REUNIÃO BRASILEIRA DE FERTILIDADE DO SOLO E NUTRIÇÃO DE PLANTAS, 22., 1996, Manaus. [Palestras...]. Manaus: Ed. da Universidade do Amazonas, 1996. p. 238-239. 
GOMES, K. C. O. de; PAIVA, H. N. de; NEVES, J. C. L.; BARROS, N. F.de; SILVA, S. R. Influência da saturação por bases e do fósforo no crescimento de mudas de angico-branco. Revista Árvore, Viçosa, MG, v. 28, n. 6, p. 785-792, 2004.

GONÇALVES, J. L. M.; SANTARELLI, E. G.; MORAES NETO, S. P.; MANARA, M. P. Produção de mudas de espécies nativas: substrato, nutrição, sombreamento e fertilização. In: GONÇALVES, J. L. M.; BENEDETTI, V. (Eds.). Nutrição e fertilização florestal. Piracicaba: IPEF, 2000. 427 p.

LAMPRECHT, H. Silvicultura nos trópicos: ecossistemas florestais e respectivas espécies arbóreas: possibilidades e métodos de aproveitamento sustentado. Traduação de: Guilherme de Almeida-Sedas: Gilberto. Eschoborn: GTZ, 1990. 343 p.

LOUREIRO, A. A.; SILVA, M. F.; ALENCAR, J. C. Essências madeireiras da Amazônia. Manaus: Instituto Nacional de Pesquisas da Amazônia.. 1979. v. 2.

MALAVOlTA, E. Manual de química agrícola: adubos e adubações. 3. ed. São Paulo: Ceres. 1981. $596 \mathrm{p}$.

MAlavolta, E.; VITTI, G. C.; OLIVEIRA, S. A. Avaliação do estado nutricional de plantas: princípios e aplicações. Piracicaba: POTAFOS. 1989. 201 p.

MANN, E. N.; FURTINI NETO, A. E.; RESENDE, A. V.; VALE, F. R; FONSECA, F. C. Calagem e crescimento de espécies florestais. In: REUNIÃO BRASILEIRA DE FERTILIDADE DO SOLO E NUTRIÇÃO DE PLANTAS, 22., 1996, Manaus. [Palestras...]. Manaus: Ed. da Universidade do Amazonas, 1996.. 240-241.

NOVAIS, R. F.; NEVES, J. C. L.; BARROS, N. F. Fósforo. In: Curso de fertilidade e manejo de solo. BRASÍLIA: ABEAS, 1994. 133 p.

OSAKI, F. Calagem e adubação. 2. ed. Campinas: Instituto Brasileiro de Ensino Agrícola. 1991. 503 p.

QUAGGIO, J. A. Reação do solo e seu controle. In: Simpósio Avançado de Química e Fertilidade do Solo. Fundação Cargill. 1986. 179 p.

RAMOS, M. R. C. Crescimento e nutrição mineral de mudas de pata-de-vaca (Bauhinia forficata Link). 41 f. Dissertação (Mestrado em Fitotecnia) - Universidade Federal de Lavras, Lavras, 1999.

RIBEIRO, A. C.; GUIMARÃES, P. T. G.; AlVAREZ, V. V. H. (Eds). Recomendação para uso de corretivos e fertilizantes no Estado de Minas Gerais: $5^{\mathrm{a}}$ Aproximação. Viçosa, MG: Comissão de Fertilidade de Solo do Estado de Minas Gerais, 1999. 359 p.

RIZZINI, C. T. Árvores e madeiras úteis do Brasil: manual de dendrologia. 2. ed. São Paulo: E. Blucher, 1990. 286 p.

RESENDE, A. V. Nutrição e crescimento de espécies florestais de diferentes grupos sucessionais em resposta à fertilização fosfatada na fase de mudas. 76 f. Dissertação (Mestrado em Solos) Universidade Federal de Lavras, Lavras, 1997.

SILVA, A. R. M. da; TUCCI, C. A. F.; LIMA, A. F. F.; FIGUEIREDO, A. F. Doses crescentes de corretivo na formação de mudas de mogno (Swietenia macrophylla King). Acta Amazônica, Manaus, v. 3, n. 2, p.195-200, 2007.

SOUSA, H. U. Crescimento e nutrição mineral de mudas de açaizeiro (Euterpe oleraceae Mart.). 124 f. Tese (Doutorado em Agronomia) - Universidade Federal de Lavras, Lavras,, 2000.

TEDESCO, N. Produção de mudas de Acácia-negra (Acacia mearnsii De Wild.) adubadas com NPK. 71 f. Dissertação (Mestrado em Engenharia Florestal) - Universidade Federal de Santa Maria, Santa Maria, RS, 1999.

TUCCI, C. A. F. Estimativa da necessidade de calagem para solos da Amazônia. 46 f. Tese (Professor Titular) - Universidade Federal do Amazonas, Manaus, 1996. 
TUCCI, C. A. F.; HARA, F. A. S.; FREITAS, R. O. de. Adubação e calagem para a formação de mudas de sumaúma (Ceiba pentandra (L.) Gaertn). Revista de Ciências Agrárias e Ambientais da UFAM, Manaus, v.11, n. 2/2, jan., 2004.

VITTI, G. C.; MALAVOLTA, E.; COUTINHO, E. L. M. Uso eficiente de fertilizantes nitrogenados e portadores de enxofre. In: SIMPÓSIO SOBRE FERTILIZANTES NA AGRICULTURA BRASILEIRA, 1984, Brasília, DF. Anais... Brasília, DF: EMBRAPA - DEP, 1984. 641 p. 